\title{
REMARKS ON SOME MODULAR IDENTITIES
}

\author{
BY \\ MORRIS NEWMAN
}

Introduction. We shall consider a certain class of functions invariant with respect to the substitutions of the congruence subgroup $\Gamma_{0}(p)$ of the modular group $\Gamma$. By specializing these functions, we shall obtain classical identities in the analytical theory of numbers: E.g., the Ramanujan identities for partitions modulo 5, 7 and Mordell's identity for $\tau(n)$. We shall also derive some new identities.

These functions bear some resemblance to those considered by Rademacher in his paper [1]( $\left.{ }^{1}\right)$ to prove the Ramanujan identities, certain modular equations, etc. The type of function considered, however, seems first to have been studied by Watson in his paper [2].

\section{Definitions, notations.}

(1.1) $\Gamma$ is the full modular group; i.e., the group of $2 \times 2$ matrices of determinant 1 with rational integral elements.

(1.2) $\Gamma_{0}(m)$ is the subgroup of $\Gamma$ characterized as follows: The element

$$
\left(\begin{array}{ll}
a & b \\
c & d
\end{array}\right)
$$

of $\Gamma$ belongs to $\Gamma_{0}(m)$ if and only if $c \equiv 0(m)$.

$$
\begin{aligned}
\eta(\tau) & =\exp \pi i \tau / 12 \cdot \prod\left(1-x^{n}\right) \\
& =\exp \pi i \tau / 12 \cdot\left\{\sum p(n) x^{n}\right\}^{-1}, \quad x=\exp 2 \pi i \tau, i m \tau>0 .
\end{aligned}
$$

Here, as in the sequel, all products will be extended from 1 to $\infty$ and all sums from 0 to $\infty$, unless otherwise indicated.

A few words about $\eta(\tau)$ (the Dedekind $\eta$-function) are in order. In the interior of the upper half-plane, $\eta(\tau)$ is free from poles and zeros. At $\tau=i \infty$ and at $\tau=0, \eta(\tau)$ is zero. $\eta(\tau)$ is a modular form of dimension $-1 / 2$, and satisfies the following transformation formula, which will be used extensively:

(1.4) If

$$
V=\left(\begin{array}{ll}
a & b \\
c & d
\end{array}\right) \in \Gamma, \quad \text { and } \quad c>0
$$

then

$$
\eta(V \tau)=\{-i(c \tau+d)\}^{1 / 2} \exp -\pi i N \cdot \eta(\tau),
$$

Received by the editors December 6, 1951 .

(1) Numbers in brackets refer to bibliography at end of paper. 
where $N=s(a, c)-(a+d) / 12 c$. Also, $\eta(\tau+1)=\exp \pi i / 12 \cdot \eta(\tau)$.

$s(a, c)$ is a "Dedekind sum" and is defined as follows: If we set

$$
((x))=\left\{\begin{array}{cl}
x-[x]-1 / 2 & x \text { nonintegral } \\
0 & x \text { integral }
\end{array}\right.
$$

then $s(a, c)=\sum((r / c))((a r / c))$, where $r$ runs over a complete set of residues modulo $c$ in the summation.

We shall put

$$
S=\left(\begin{array}{ll}
1 & 1 \\
0 & 1
\end{array}\right), \cdot \quad T=\left(\begin{array}{rr}
0 & -1 \\
1 & 0
\end{array}\right), \quad W=\left(\begin{array}{ll}
1 & 0 \\
1 & 1
\end{array}\right) .
$$

It is well known that $S$ and $T$ are generators of $\Gamma$. We observe that

$$
\begin{gathered}
S^{n}=\left(\begin{array}{ll}
1 & n \\
0 & 1
\end{array}\right), \\
W^{n}=\left(\begin{array}{ll}
1 & 0 \\
n & 1
\end{array}\right), \quad T^{2}=-I .
\end{gathered}
$$

Since

$$
\left(\begin{array}{ll}
a & b \\
c & d
\end{array}\right) \tau=(a \tau+b) /(c \tau+d)=\left(\begin{array}{ll}
-a & -b \\
-c & -d
\end{array}\right) \tau
$$

$T^{2} \tau=\tau$.

We shall also put

$$
z_{p}=\exp -2 \pi i / p \tau, \quad \Pi\left(1-x^{n}\right)^{r}=\sum p_{r}(n) x^{n} .
$$

We shall be concerned with series whose coefficients are $p_{r}(n)$ 's.

Notice that $p_{-1}(n)=p(n)$ in this notation.

Some remarkable congruence properties of the $p_{r}(n)$ 's modulo 5,7 have been found by Ramanathan in his paper [6].

2. Functions on $\Gamma_{0}(p)$. In what follows, $p$ will always be a prime greater than 3. The fundamental region $Q_{p}$ of $\Gamma_{0}(p)$ has only the parabolic points $i \infty, 0$. A function on $\Gamma_{0}(p)$ (invariant with respect to the substitutions of $\left.\Gamma_{0}(p)\right)$ which is regular in the interior of $Q_{p}$ and bounded at $i \infty, 0$ accordingly is constant. For this reason functions on $\Gamma_{0}(p)$ are amenable to numerical calculation. We need the following elementary theorem, the proof of which we omit:

(2.1) $\Gamma_{0}\left(p^{2}\right)$ is of index $p$ in $\Gamma_{0}(p)$, and a set of right representatives for $\Gamma_{0}\left(p^{2}\right)$ in $\Gamma_{0}(p)$ is given by:

$$
R_{k}=W^{-p k}, \quad k=0,1, \cdots, p-1 .
$$

We also need the following: 
(2.2) Theorem. Let $\Gamma_{0}, \Gamma_{1}$ be subgroups of $\Gamma, \Gamma_{0} \supseteq \Gamma_{1},\left(\Gamma_{0}: \Gamma_{1}\right)=\mu<\infty$. Let $R_{0}, R_{1}, \cdots, R_{\mu-1}$ be a set of right representatives for $\Gamma_{1}$ in $\Gamma_{0}$. Let $g(\tau)$ be a function on $\Gamma_{1}$, and let $F\left(x_{0}, x_{1}, \cdots, x_{\mu-1}\right)$ be a symmetric function of its variables. Put $f(\tau)=F\left(g\left(R_{0} \tau\right), g\left(R_{1} \tau\right), \cdots, g\left(R_{\mu-1} \tau\right)\right)$. Then $f(\tau)$ is a function on $\Gamma_{0}$.

Proof. Let $M \in \Gamma_{0}$.

$R_{i} M$ may be written as $M_{i} R_{i}^{\prime}$, where $M_{i} \in \Gamma_{1}$, and $R_{i}^{\prime}$ is a representative. Also, if $R_{i} M=M_{i} R_{i}^{\prime}, R_{j} M=M_{j} R_{j}^{\prime}$, then if $R_{i}^{\prime}=R_{j}^{\prime}$, we have $R_{i} R_{j}^{-1}=M_{i} M_{j}^{-1}$ $\in \Gamma_{1}$, so that $R_{i}=R_{j}$. That is, as $R_{i}$ runs through a complete set of representatives, so does $R_{i}^{\prime}$.

Hence

$$
\begin{aligned}
f(M \tau) & =F\left(g\left(R_{0} M \tau\right), g\left(R_{1} M \tau\right), \cdots, g\left(R_{\mu-1} M \tau\right)\right) \\
& =F\left(g\left(M_{0} R_{0}^{\prime} \tau\right), g\left(M_{1} R_{1}^{\prime} \tau\right), \cdots, g\left(M_{\mu-1} R_{\mu-1}^{\prime} \tau\right)\right) \\
& =F\left(g\left(R_{0}^{\prime} \tau\right), g\left(R_{1}^{\prime} \tau\right), \cdots, g\left(R_{\mu-1}^{\prime} \tau\right)\right) \\
& =f(\tau) .
\end{aligned}
$$

The function $\eta\left(p^{2} \tau\right) / \eta(\tau)=x^{\nu} \prod\left\{\left(1-x^{p^{2} n}\right) /\left(1-x^{n}\right)\right\}$, where $\nu=\left(p^{2}-1\right) / 24$ (which is an integer since $(p, 6)=1$ ), may easily be shown to be a function on $\Gamma_{0}\left(p^{2}\right)$ by use of the transformation formula (1.4) and some theorems about $s(a, c)$ which may be found in [1] or in [3]. We shall omit the proof. We put

$$
\begin{aligned}
h & =h(\tau)=\eta\left(p^{2} \tau\right) / \eta(\tau), \\
S_{r} & =S_{r}(\tau)=\sum_{n=0}^{p-1} h^{r}\left(R_{n} \tau\right), r \text { integral. }
\end{aligned}
$$

By (2.1) and (2.2), $S_{r}$ is a function on $\Gamma_{0}(p)$. The $S_{r}$ 's are the functions we shall consider. Clearly, $S_{r}$ is regular and bounded in the interior of the upper half-plane (see the remark to (1.3)). Hence to determine the behaviour of $S_{r}$ completely, we need only know its behaviour at the parabolic points $i \infty, 0$ of $Q_{p}$. It will be our purpose in general to compare $S_{r}$ with

$$
g=g(\tau)=\{\eta(p \tau) / \eta(\tau)\}^{s},
$$

where $s=s(p)$ is the least positive even integer such that $s(p-1) \equiv 0(24)$. $g$ is also a function on $\Gamma_{0}(p)$ (see [1]). $g$ has a zero of order $s(p-1) / 24$ at $\tau=i \infty$ in $x$, and a pole of order $s(p-1) / 24$ at $\tau=0$ in $z_{p}$. For

$$
\begin{array}{lll}
p=5 & p=7 & p=13 \\
s=6, & s=4, & s=2,
\end{array}
$$

$g$ is a "Hauptmodul" for $\Gamma_{0}(p)$ : I.e., any function on $\Gamma_{0}(p)$ with polar singularities at most in appropriate uniformizing variables is a rational function of $g$.

We shall rewrite $S_{r}$. We have 
$h\left(R_{n} \tau\right)=\eta\left(p^{2} R_{n} \tau\right) / \eta\left(R_{n} \tau\right)=\eta\left(p^{2} W^{-p n} \tau\right) / \eta\left(W^{-p n} \tau\right)=\eta\left(p W^{-n} p \tau\right) / \eta\left(W^{-p n} \tau\right)$.

For $(n, p)=1$ define $n^{\prime}$ as the least positive solution of the congruence $n x \equiv 1(p)$, and for $p \mid n$ define $n^{\prime}=0$.

If $(n, p)=1$, we may rewrite $p W^{-n} p \tau$ as follows:

$$
p W^{-n} p \tau=M_{1}\left(\tau-n^{\prime} / p\right), \quad \text { where } M_{1}=\left(\begin{array}{cc}
-p & -n^{\prime} \\
n & \left(n n^{\prime}-1\right) / p
\end{array}\right) .
$$

Hence $h\left(R_{n} \tau\right)=\eta\left(M_{1}\left(\tau-n^{\prime} / p\right)\right) / \eta\left(W^{-p n} \tau\right)$. Making use of (1.4), we obtain $h\left(R_{n} \tau\right)=p^{-1 / 2} \exp -\pi i N_{1} \cdot \eta\left(\tau-n^{\prime} / p\right) / \eta(\tau)$, where

$$
N_{1}=\left\{s(-p, n)-\left(-n+\left(n n^{\prime}-1\right) / p\right) / 12 n\right\}-\{s(-1, p n)+1 / 6 p n\} .
$$

It may further be shown by theorems from [1] and [3] that exp $-\pi i N_{1}$ $=\exp -\pi i(p-1) / 4 \cdot \exp \pi i n^{\prime} p / 12 \cdot\left(n^{\prime} / p\right)$, where $\left(n^{\prime} / p\right)$ denotes the LegendreJacobi symbol of quadratic reciprocity. Hence

$$
h\left(R_{n} \tau\right)=p^{-1 / 2} \exp -\pi i(p-1) / 4 \cdot \exp \pi i n^{\prime} p / 12 \cdot\left(n^{\prime} / p\right) \cdot \eta\left(\tau-n^{\prime} / p\right) / \eta(\tau),
$$

and so

$$
\begin{aligned}
S_{r} & =\sum_{n=0}^{p-1} h^{r}\left(R_{n} \tau\right)=h^{r}(\tau)+\sum_{n=1}^{p-1} h^{r}\left(R_{n} \tau\right) \\
& =h^{r}(\tau)+c^{r} \eta^{-r}(\tau) \sum_{n=1}^{p-1}\left(n^{\prime} / p\right)^{r} \cdot \exp \pi i n^{\prime} p r / 12 \cdot \eta^{r}\left(\tau-n^{\prime} / p\right),
\end{aligned}
$$

where we have put $c=p^{-1 / 2} \exp -\pi i(p-1) / 4$.

Distinguishing cases $r$ even and $r$ odd, and using the well known Gaussian sum formula

$$
\sum_{n=1}^{p-1}(n / p) \exp 2 \pi i n a / p=\exp \pi i(p-1)^{2} / 8 \cdot p^{1 / 2}(a / p),
$$

we find

(2.5.1) For $r$ even,

$$
S_{r}=-c^{r}+h^{r}(\tau)+p c^{r} \Pi\left(1-x^{n}\right)^{-r} \sum_{\lambda \geqq 0, \lambda \equiv \equiv_{r \nu}(p)} p_{r}(\lambda) x^{\lambda} .
$$

(2.5.2) For $r$ odd,

$$
S_{r}=h^{r}(\tau)+\exp \pi i(p-1)^{2} / 8 \cdot p^{1 / 2} c^{r} \Pi\left(1-x^{n}\right)^{-r} \sum_{\lambda \geqq 0}((r \nu-\lambda) / p) p_{r}(\lambda) x^{\lambda} \text {. }
$$

The formulae (2.5.1), (2.5.2) furnish the desired information as to the behaviour of the $S_{r}$ 's at $i \infty$. To study the behaviour of the $S_{r}$ 's at 0 , we subject them to the transformation $T$ and study the $T S_{r}$ 's at $i \infty\left(^{2}\right)$. Proceeding

(2) A device employed by Rademacher in [1]. 
as before, we have for $h\left(R_{n} T \tau\right),(n, p)=1$ :

$$
h\left(R_{n} T \tau\right)=c\left(n^{\prime} / p\right) \exp \pi i n^{\prime} p / 12 \cdot \eta\left(T \tau-n^{\prime} / p\right) / \eta(T \tau) .
$$

We may rewrite $T \tau-n^{\prime} / p$ as follows:

$$
T \tau-n^{\prime} / p=M_{2}(\tau+n p) / p^{2}, \quad \text { where } M_{2}=\left(\begin{array}{cc}
-n^{\prime} & \left(n n^{\prime}-1\right) / p \\
p & -n
\end{array}\right) .
$$

Making use of (1.4) again,

$$
\begin{aligned}
h\left(R_{n} T \tau\right) & =c\left(n^{\prime} / p\right) \exp \pi i n^{\prime} p / 12 \cdot \eta\left(M_{2}(\tau+n p) / p^{2}\right) / \eta(T \tau) \\
& =p^{-1 / 2} c\left(n^{\prime} / p\right) \exp \pi i n^{\prime} p / 12 \cdot \exp -\pi i N_{2} \cdot \eta\left((\tau+n p) / p^{2}\right) / \eta(\tau),
\end{aligned}
$$

where $N_{2}=s\left(-n^{\prime}, p\right)+\left(n+n^{\prime}\right) / 12 p$.

After the necessary simplifications, we obtain

$$
h\left(R_{n} T \tau\right)=p^{-i} \exp -\pi i n p / 12 \cdot \eta\left(\frac{\tau+n p}{p^{2}}\right) / \eta(\tau) .
$$

If we note here that $h\left(R_{n} T \tau\right)$ agrees formally with $h(T \tau)$ for $n=0$, we have

$$
\begin{aligned}
T S_{r} & =\sum_{n=0}^{p-1} h^{r}\left(R_{n} T \tau\right) \\
& =p^{-r} \eta^{-r}(\tau) \sum_{n=0}^{p-1} \exp -\pi i n p r / 12 \cdot \eta^{r}\left(\frac{\tau+n p}{p^{2}}\right) / \eta(\tau) .
\end{aligned}
$$

Expanding into a power series, we have

$$
T S_{r}=p^{-r+1} \prod\left(1-x^{n}\right)^{-r} \sum_{\lambda \geqq 0, \lambda \equiv r \nu(p)} p_{r}(\lambda) x^{(\lambda-r \nu) / p^{2}} .
$$

A study of (2.5.1), (2.5.2), and (2.7) leads us to the construction of the following table:

(2.8) (a) $r>0$.

$$
i \infty
$$

0

$S_{r}: \quad$ Zero-free and pole-free in $x$ unless $r$ is odd and Pole of order

$S_{r}+c^{r}$ divisible by $p$, in which case zero of order 1 in $x$.

$r$ even: Zero of order $r \nu-p[r \nu / p]$ in $x\left(^{3}\right)$.

(b) $r<0$. Put $r=-r_{1}, r_{1}>0$. We then have:

$$
i \infty
$$

$S_{r}: \quad$ Pole of order $r_{1} \nu$ in $x$.

$[r \nu / p]$ in $z_{p}\left({ }^{3}\right)$. Pole of order $[r \nu / p]$ in $z_{p}\left({ }^{3}\right)$.

0

Zero of order $1+\left[r_{1} \nu / p\right]$ in $z_{p}$.

\section{Applications.}

(3) Provided that $p_{r}(r \nu-p[r \nu / p]) \neq 0$. 
(3.1) $r=-1, p=5$.

Then $S_{-1}$ has a pole of order 1 in $x$ at $\tau=i \infty$, and a zero of order 1 in $z_{5}$ at $\tau=0$. This implies that the product $g S_{-1}$ is constant, or that

$$
S_{-1}=K_{0} g^{-1} \text {. }
$$

If we replace $\tau$ by $-1 / 5 \tau$ and evaluate $K_{0}$, we obtain the first of Ramanujan's identities:

$$
\sum p(5 n+4) x^{n}=5 \prod\left(1-x^{5 n}\right)^{5}\left(1-x^{n}\right)^{-6} .
$$

(3.2) $r=-1, p=7$.

Then $S_{-1}$ has a pole of order 2 in $x$ at $\tau=i \infty$, and a zero of order 1 in $z_{7}$ at $\tau=0$. This implies that $g^{2} S_{-1}$ is linear in $g$, or that

$$
S_{-1}=K_{0} g^{-2}+K_{1} g^{-1} \text {. }
$$

If we replace $\tau$ by $-1 / 7 \tau$ and evaluate $K_{0}, K_{1}$, we obtain the second of Ramanujan's identities:

$$
\sum p(7 n+5) x^{n}=7 I\left(1-x^{7 n}\right)^{3}\left(1-x^{n}\right)^{-4}+49 x \prod\left(1-x^{7 n}\right)^{7}\left(1-x^{n}\right)^{-8} \text {. }
$$

(3.3) $r=-1, p=13$.

Then $S_{-1}$ has a pole of order 7 in $x$ at $\tau=i \infty$, and a zero of order 1 in $z_{13}$ at $\tau=0$. This implies that $g^{7} S_{-1}$ is a polynomial in $g$ of degree 6 , or that

$$
S_{-1}=K_{0} g^{-7}+K_{1} g^{-6}+\cdots+K_{6} g^{-1} \text {. }
$$

If we replace $\tau$ by $-1 / 13 \tau$ and evaluate $K_{0}, K_{1}, \cdots, K_{6}$, we obtain an identity of Zuckerman (see [3]).

(3.4) $r=24$.

Then $S_{24}+c^{24}$ has a zero of order $p-1$ at least in $x$ at $\tau=i \infty$, and a pole of order $p-1$ at most in $z_{p}$ at $\tau=0$. This implies that the quotient $\left(S_{24}+c^{24}\right) /\{\eta(p \tau) / \eta(\tau)\}^{24}$ is bounded, which in turn implies that

$$
S_{24}=-c^{24}+K_{0}\{\eta(p \tau) / \eta(\tau)\}^{24} .
$$

If we replace $\tau$ by $-1 / p \tau$, evaluate $K_{0}$, and set $\tau(n)=p_{24}(n-1)$, we obtain Mordell's identity for $\tau(n)\left({ }^{4}\right)$ :

$$
\sum \tau(n p+p) x^{n}=\tau(p) \Pi\left(1-x^{n}\right)^{24}-p^{11} x^{p-1} \Pi\left(1-x^{n p}\right)^{24} .
$$

(3.5) We can easily generalize Mordell's identity as follows: Choose $r$ even, $0<r \leqq 24, r(p-1) \equiv 0$ (24). Put $\delta=r(p-1) / 24$. Then we have for $S_{r}+c^{r}$ :

$$
i \infty \quad 0
$$

$S_{r}+c^{r}: \quad$ Zero of order $\delta$ at least in $x$. Pole of order $\delta$ at most in $z_{p}$.

This implies that the quotient $\left(S_{r}+c^{r}\right) /\{\eta(p \tau) / \eta(\tau)\} r$ is bounded which (4) See [5]. 
in turn implies that

$$
S_{r}=-c^{r}+K_{0}\{\eta(p \tau) / \eta(\tau)\} r .
$$

If we replace $\tau$ by $-1 / p \tau$ and evaluate $K_{0}$, we obtain

$$
\sum p_{r}(n p+\delta) x^{n}=p_{r}(\delta) \prod\left(1-x^{n}\right)^{r}-p^{r / 2-1} x^{\delta} \prod\left(1-x^{n p}\right)^{r} .
$$

(3.6) $p=5$.

We have the following table:

$i \infty$

$S_{1}: \quad$ Zero-free and pole-free in $x$.

$S_{2}$ : Zero-free and pole-free in $x$.

$S_{3}: \quad$ Zero-free and pole-free in $x$.

$S_{4}: \quad$ Zero-free and pole-free in $x$.

$S_{5}$ : Zero of order 1 in $x$.
0

Zero-free and pole-free in $z_{5}$. Zero-free and pole-free in $z_{5}$. Zero-free and pole-free in $z_{5}$. Zero-free and pole-free in $z_{5}$. Pole of order 1 in $z_{5}$.

These imply that $S_{1}, S_{2}, S_{3}, S_{4}$ are constant, while $S_{5}$ is proportional to $B=\{\eta(5 \tau) / \eta(\tau)\}^{6}$. Hence in the polynomial $\prod_{i=0}^{4}\left(u-h\left(R_{i} \tau\right)\right)=u^{5}-c_{1} u^{4}$ $+c_{2} u^{3}-c_{3} u^{2}+c_{4} u-c_{5}$, the coefficients $c_{1}, c_{2}, c_{3}, c_{4}$ must also be constant, while $c_{5}$ is linear in $B\left({ }^{5}\right)$.

Putting $u=h(\tau)=\eta(25 \tau) / \eta(\tau)=A$, we find that $B$ is a polynomial of degree 5 in $A$. The actual polynomial turns out to be

$$
B=25 A^{5}+25 A^{4}+15 A^{3}+5 A^{2}+A .
$$

If we set $A_{0}=5^{1 / 2} A, B_{0}=B / A$, then (3.6.1) reads

$$
B_{0}=A_{0}^{4}+5^{1 / 2} A_{0}^{3}+3 A_{0}^{2}+5^{1 / 2} A_{0}+1 .
$$

(3.6.2) shows the reciprocal nature of the modular equation.

The reason there is an identity (3.6.1) is that $\Gamma_{0}(25)$ is of genus zero (as are $\Gamma_{0}(5), \Gamma_{0}(7), \Gamma_{0}(13)$ ) and so a Hauptmodul exists for $\Gamma_{0}(25)$. (3.6.1) indicates that we may choose $\eta(25 \tau) / \eta(\tau)$ for this Hauptmodul( $\left.{ }^{6}\right)$. The genus of $\Gamma_{0}(49)$, however, turns out to be 1 , so no analogous identity exists for $\{\eta(7 \tau) / \eta(\tau)\}^{4}$ in terms of $\eta(49 \tau) / \eta(\tau)$. The polynomial relationship between these functions is quadratic in $\{\eta(7 \tau) / \eta(\tau)\}^{4}$.

Some new identities (of the many which are possible) obtained by specializing the $S_{r}$ 's follow, without comment. Of particular interest are those for which $p$ differs from $5,7,13$ (since then the subgroups in question are not of genus zero). These identities are isolated instances, not at all characteristic

${ }^{(5)}$ The $c$ 's are the elementary symmetric functions on the roots, and the result follows from Newton's formulae relating the c's and the $S$ 's.

(6) $\eta(25 \tau) / \eta(\tau)$ has a zero of order 1 in $x$ at $\tau=i \infty$, a pole of order 1 in $z_{25}$ at $\tau=0$, and is finite and different from zero at the other parabolic points $\pm 1 / 5, \pm 2 / 5$ of $Q_{25}$. That is, it has precisely one pole (in the proper uniformizing variable) in $Q_{25}$. This guarantees that it is a Hauptmodul for $\Gamma_{0}(25)$. 
of the true situation.

1. $r=5, p=5$.

$\sum p_{5}(5 n) x^{n}=\prod\left(1-x^{n}\right)^{6}\left(1-x^{5 n}\right)^{-1}(7)$.

2. $r=5, p=7$.

$\sum p_{5}(7 n+3) x^{n}=10 \prod\left(1-x^{7 n}\right)\left(1-x^{n}\right)^{4}+49 x \prod\left(1-x^{7 n}\right)^{5}$.

3. $r=7, p=7$.

$\sum p_{7}(7 n) x^{n}=\prod\left(1-x^{n}\right)^{8}\left(1-x^{7 n}\right)^{-1}+49 x \prod\left(1-x^{n}\right)^{4}\left(1-x^{7 n}\right)^{3}(7)$.

4. $r=2, p=11$.

$\sum p_{2}(11 n+10) x^{n}=\prod\left(1-x^{11 n}\right)^{2}$.

5. $r=4, p=11$.

$\sum p_{4}(11 n+20) x^{n}=-11 \prod\left(1-x^{11 n}\right)^{4}$.

6. $r=2, p=17$.

$\sum p_{2}(17 n+24) x^{n}=-\prod\left(1-x^{17 n}\right)^{2}$.

7. $r=6, p=31$.

$\sum p_{6}(31 n+240) x^{n}=961 \prod\left(1-x^{31 n}\right)^{6}$.

\section{BIBLIOGRAPHY}

1. H. A. Rademacher, The Ramanujan identities under modular substitutions, Trans. Amer. Math. Soc. vol. 51 (1942) pp. 609-636.

2. G. N. Watson, Ramanujan's Vermutung ïber Zerfallungs anzahlen, J. Reine Angew. Math. vol. 179 (1938) pp. 97-128.

3. H. S. Zuckerman, Identities analogous to Ramanujan's identities involving the partition function, Duke Math. J. vol. 5 (1939) pp. 88-110.

4. H. A. Rademacher and A. Whiteman, Theorems on Dedekind sums, Amer. J. Math. vol. 63 (1941) pp. 377-407.

5. G. H. Hardy, Ramanujan, Cambridge University Press, 1940, pp. 161-163.

6. K. G. Ramanathan, Identities and congruences of the Ramanujan type, Canadian Journal of Mathematics vol. 2 (1950) p. 168.

National Bureau of Standards, WASHINGTON, D. C.

(7) Dr. Lehmer informs me that these identities are known to him, though he has never published them. 